\title{
Blow-Up and Global Existence for a Quasilinear Parabolic System
}

\author{
Chunchen Wu \\ Zhicheng College, Fuzhou University, Fuzhou, Fujian 350002, China \\ Correspondence should be addressed to Chunchen Wu; 237621488@qq.com \\ Received 22 October 2013; Accepted 5 January 2014; Published 13 February 2014 \\ Academic Editor: Elena Braverman \\ Copyright (C) 2014 Chunchen Wu. This is an open access article distributed under the Creative Commons Attribution License, which \\ permits unrestricted use, distribution, and reproduction in any medium, provided the original work is properly cited. \\ The problem of solutions to a class of quasilinear coupling parabolic system was studied. By constructing weak upper-solutions and \\ weak lower-solutions, we obtain the global existence and blow-up of solutions under appropriate conditions.
}

\section{Introduction and Main Result}

In this paper, we consider global existence of nonnegative solutions for a class of nonlocal degenerate quasilinear parabolic system as follows:

$$
\begin{gathered}
u_{t}=\Delta u^{m}+u^{p_{1}}\|v\|_{\alpha}^{p_{2}}, \\
v_{t}=\Delta v^{n}+v^{q_{1}}\|w\|_{\beta}^{q_{2}}, \\
w_{t}=\Delta w^{h}+w^{r_{1}}\|u\|_{\gamma}^{r_{2}}, \\
(x, t) \in \Omega \times(0, T), \\
u(x, t)=v(x, t)=w(x, t)=0, \quad(x, t) \in \partial \Omega \times(0, T), \\
u(x, 0)=u_{0}(x), \\
v(x, 0)=v_{0}(x), \\
w(x, 0)=w_{0}(x), \\
x \in \Omega,
\end{gathered}
$$

where $\Omega \in R^{N}$ is bounded region; $\Omega$ has smooth boundary $\partial \Omega$; the parameters $m, n, h>1, \alpha, \beta, \gamma \geq 1, p_{1}, q_{1}$, and $r_{1} \geq 0, p_{2}, q_{2}, r_{2}>0$; the initial functions $u_{0}, v_{0}$, and $w_{0}$ are nonnegative and bounded, and $\|\cdot\|_{\alpha}^{\alpha}=\int_{\Omega}|\cdot|^{\alpha} d x$.

Quasilinear parabolic system is the model for many problems in the scientific field, for example, gas flow model in some seepage medium, some biological population growth model. In recent years, there are many papers to investigate the nonlinear parabolic equation and many excellent results are obtained (see [1-10] and the references cited therein). In this paper, we expand the equation of [10] into 3 and discuss the global existence and blow-up of the solutions for problem (1), and the main results of this paper are the following.

Theorem 1. If one of the following conditions holds, system (1) has global solutions:

(1) $m>p_{1}, n>q_{1}, h>r_{1}$, and $p_{2} q_{2} r_{2}<\left(m-p_{1}\right)(n-$ $\left.q_{1}\right)\left(h-r_{1}\right)$

(2) $m>p_{1}, n>q_{1}, h>r_{1}$, and $p_{2} q_{2} r_{2}=(m-$ $\left.p_{1}\right)\left(n-q_{1}\right)\left(h-r_{1}\right)$, and the magnitude of the region $\Omega$ is sufficiently small;

(3) If $m \leq p_{1}, n \leq q_{1}$, or $h \leq r_{1}$, or if $m>p_{1}, n>q_{1}, h>$ $r_{1}$, and $p_{2} q_{2} r_{2}>\left(m-p_{1}\right)\left(n-q_{1}\right)\left(h-r_{1}\right)$, and the initial data $u_{0}, v_{0}$, and $w_{0}$ are sufficiently small.

Theorem 2. If one of the following conditions holds, the solution of system (1) blows up in finite time:

(1) $m>p_{1}, n>q_{1}, h>r_{1}$, and $p_{2} q_{2} r_{2}>\left(m-p_{1}\right)(n-$ $\left.q_{1}\right)\left(h-r_{1}\right)$, and the initial data $u_{0}, v_{0}$, and $w_{0}$ are sufficiently large;

(2) $m \leq p_{1}, n \leq q_{1}$, or $h \leq r_{1}$, and the initial data $u_{0}, v_{0}$, and $w_{0}$ are sufficiently large.

\section{Proof of Global Existence}

As we know, nonlocal degenerate quasilinear parabolic system may not have classical solutions. Similar to the proof in [10] (see page 388-389), we can obtain that system (1) 
has nonnegative weak upper-solutions and nonnegative weak lower-solutions, and the following comparison principle holds.

Lemma 3 (comparison principle). Suppose $(\bar{u}, \bar{v}, \bar{w}),(\underline{u}, \underline{v}, \underline{w})$ are the nonnegative weak upper-solutions and nonnegative weak lower-solutions of system (1), if

$$
\bar{u}, \bar{v}, \bar{w} \geq \delta>0, \quad \text { or } \quad \underline{u}, \underline{v}, \underline{w} \geq \delta>0,
$$

one has $(\underline{u}(x, t), \underline{v}(x, t), \underline{w}(x, t)) \leq(\bar{u}(x, t), \bar{v}(x, t), \bar{w}(x, t))$ for every $(x, \bar{t}) \in \Omega \times(0, T)$.

Therefore, in order to prove Theorem 1, we only show that, for all $T>0$, there exists a positive bounded weak upper-solutions. Let $\varphi(x)$ be the unique positive solution of linear elliptic equation as follows:

$$
-\Delta \varphi=1, \quad x \in \Omega ; \quad \varphi(x)=0, \quad x \in \partial \Omega .
$$

Denote $C=\max _{x \in \Omega} \varphi(x)$, then $0 \leq \varphi(x) \leq C$. Define $\bar{u}(x, t), \bar{v}(x, t), \bar{w}(x, t)$ as follows:

$$
\begin{gathered}
\bar{u}=(k(\varphi(x)+1))^{l_{1}}, \quad \bar{v}=(k(\varphi(x)+1))^{l_{2}}, \\
\bar{w}=(k(\varphi(x)+1))^{l_{3}},
\end{gathered}
$$

where $l_{1}, l_{2}, l_{3}<1$ and satisfy $m l_{1}, n l_{2}, h l_{3}<1$. $k$ is a positive constant to be determined suitably. For all $T>0, \bar{u}, \bar{v}$, and $\bar{w}$ are bounded, and $\bar{u} \geq k^{l_{1}}, \bar{v} \geq k^{l_{2}}$, and $\bar{w} \geq k^{l_{3}}$. By deducing, we have

$$
\begin{gathered}
\bar{u}_{t}-\Delta \bar{u}^{m}=-k^{m l_{1}}\left[m l_{1}\left(m l_{1}-1\right)(\varphi+1)^{m l_{1}-2}|\nabla \varphi|^{2}\right. \\
\left.+m l_{1}(\varphi+1)^{m l_{1}-1} \Delta \varphi\right] \\
\geq m l_{1}(C+1)^{m l_{1}-1} k^{m l_{1}}, \\
\bar{u}^{p_{1}}\|\bar{v}\|_{\alpha}^{p_{2}}=(k(\varphi+1))^{l_{1} p_{1}}\left\|(k(\varphi+1))^{l_{2}}\right\|_{\alpha}^{p_{2}} \\
\leq k^{l_{1} p_{1}+l_{2} p_{2}}(C+1)^{l_{1} p_{1}+l_{2} p_{2}}|\Omega|^{p_{2} / \alpha} .
\end{gathered}
$$

Similarly

$$
\begin{gathered}
\bar{v}_{t}-\Delta \bar{v}^{n} \geq n l_{2}(C+1)^{n l_{2}-1} k^{n l_{2}}, \\
\bar{v}^{q_{1}}\|\bar{w}\|_{\beta}^{q_{2}} \leq k^{l_{2} q_{1}+l_{3} q_{2}}(C+1)^{l_{2} q_{1}+l_{3} q_{2}}|\Omega|^{q_{2} / \beta}, \\
\bar{w}_{t}-\Delta \bar{w}^{h} \geq h l_{3}(C+1)^{h l_{3}-1} k^{h l_{3}}, \\
\bar{w}^{r_{1}}\|\bar{u}\|_{\gamma}^{r_{2}} \leq k^{l_{3} r_{1}+l_{1} r_{2}}(C+1)^{l_{3} r_{1}+l_{1} r_{2}}|\Omega|^{r_{2} / \gamma} .
\end{gathered}
$$

Denote

$$
\begin{gathered}
C_{1}=\left(\frac{|\Omega|^{p_{2} / \alpha}}{m l_{1}}(C+1)^{l_{1} p_{1}+l_{2} p_{2}-m l_{1}+1}\right)^{1 /\left(m l_{1}-l_{1} p_{1}-l_{2} p_{2}\right)} ; \\
C_{2}=\left(\frac{|\Omega|^{q_{2} / \beta}}{n l_{2}}(C+1)^{l_{2} q_{1}+l_{3} q_{2}-n l_{2}+1}\right)^{1 /\left(n l_{2}-l_{2} q_{1}-l_{3} q_{2}\right)} ; \\
C_{3}=\left(\frac{|\Omega|^{r_{2} / \gamma}}{h l_{3}}(C+1)^{l_{3} r_{1}+l_{1} r_{2}-h l_{3}+1}\right)^{1 /\left(h l_{3}-l_{3} r_{1}-l_{1} r_{2}\right)} ;
\end{gathered}
$$

(1) If $m>p_{1}, n>q_{1}, h>r_{1}$, and $p_{2} q_{2} r_{2}<\left(m-p_{1}\right)\left(n-q_{1}\right)(h-$ $\left.r_{1}\right)$, there exist positive constants $l_{1}, l_{3}$ such that

$$
\frac{h-r_{1}}{r_{2}}>\frac{l_{1}}{l_{3}}>\frac{p_{2} q_{2}}{\left(m-p_{1}\right)\left(n-q_{1}\right)}
$$

and $m l_{1}, n l_{2}, h l_{3}<1$. And there exists positive constant $l_{2}$ such that

$$
\frac{l_{1}}{l_{2}}>\frac{p_{2}}{m-p_{1}}, \quad \frac{l_{2}}{l_{3}}>\frac{q_{2}}{n-q_{1}} .
$$

Thus,

$$
\begin{gathered}
p_{2} l_{2}<\left(m-p_{1}\right) l_{1}, \quad q_{2} l_{3}<\left(n-q_{1}\right) l_{2} \\
r_{2} l_{1}<l_{3}\left(h-r_{1}\right) .
\end{gathered}
$$

From (11), we can choose $k$ sufficiently large, such that $k>$ $\max \left\{C_{1}, C_{2}, C_{3}\right\}$ and

$$
\begin{gathered}
(k(\varphi(x)+1))^{l_{1}} \geq u_{0}(x), \quad(k(\varphi(x)+1))^{l_{2}} \geq v_{0}(x), \\
(k(\varphi(x)+1))^{l_{3}} \geq w_{0}(x) .
\end{gathered}
$$

By (4)-(12) we obtain that $(\bar{u}(x, t), \bar{v}(x, t), \bar{w}(x, t))$ is the weak upper-solutions of system (1).

(2) If $m>p_{1}, n>q_{1}, h>r_{1}$, and $p_{2} q_{2} r_{2}=\left(m-p_{1}\right)(n-$ $\left.q_{1}\right)\left(h-r_{1}\right)$, there exist positive constants $l_{1}, l_{3}$ such that

$$
\frac{h-r_{1}}{r_{2}}=\frac{l_{1}}{l_{3}}=\frac{p_{2} q_{2}}{\left(m-p_{1}\right)\left(n-q_{1}\right)} .
$$

And there exists positive constant $l_{2}$ such that

$$
\frac{l_{1}}{l_{2}}=\frac{p_{2}}{m-p_{1}}, \quad \frac{l_{2}}{l_{3}}=\frac{q_{2}}{n-q_{1}} .
$$

Therefore,

$$
p_{2} l_{2}=\left(m-p_{1}\right) l_{1}, \quad q_{2} l_{3}=\left(n-q_{1}\right) l_{2},
$$

$$
r_{2} l_{1}=l_{3}\left(h-r_{1}\right) .
$$

Without loss of generality, we assume that the domain we discuss is contained in a sufficiently large ball $B$; denote $\varphi_{B}(x)$ is the unique positive solution of the following linear elliptic equation:

$$
-\Delta \varphi=1, \quad x \in B ; \quad \varphi(x)=0, \quad x \in \partial B .
$$

Let $C_{0}=\max _{x \in B} \varphi_{B}(x)$; hence $C \leq C_{0}$. Suppose that $|\Omega|$ is sufficiently small such that

$$
|\Omega|<\min \left\{\left(\frac{m l_{1}}{C_{0}+1}\right)^{\alpha / p_{2}},\left(\frac{n l_{2}}{C_{0}+1}\right)^{\beta / q_{2}},\left(\frac{h l_{3}}{C_{0}+1}\right)^{\gamma / r_{2}}\right\} .
$$

In addition, we choose $k$ large enough, such that $k$ satisfies (12); then from (5), (6), and (12)-(17) we obtain that $(\bar{u}(x, t)$, $\bar{v}(x, t), \bar{w}(x, t))$ is the weak upper-solutions of system (1). 
(3) At last, if $m \leq p_{1}, n \leq q_{1}$, or $h \leq r_{1}$, or if $m>p_{1}$, $n>q_{1}, h>r_{1}$, and $p_{2} q_{2} r_{2}>\left(m-p_{1}\right)\left(n-q_{1}\right)\left(h-r_{1}\right)$, there exist positive constants $l_{1}, l_{2}$, and $l_{3}$ such that

$$
\begin{array}{cl}
p_{2} l_{2}>\left(m-p_{1}\right) l_{1}, & q_{2} l_{3}>\left(n-q_{1}\right) l_{2}, \\
r_{2} l_{1}>l_{3}\left(h-r_{1}\right), & m l_{1}, n l_{2}, h l_{3}<1 .
\end{array}
$$

Therefore, from (18), we can choose $k$ small enough,such that $k<\min \left\{C_{1}, C_{2}, C_{3}\right\}$. Furthermore, if $u_{0}, v_{0}$, and $w_{0}$ are sufficiently small to satisfy (12), then by (5), (6), (12), and (18) we obtain that $(\bar{u}(x, t), \bar{v}(x, t), \bar{w}(x, t))$ is the weak uppersolutions of system (1).

This completes the proof of Theorem 1.

\section{Proof of Blow-Up}

In this section we will prove Theorem 2, so we construct blow-up positive weak lower-solutions of system (1). Let $\lambda_{1}$, $\phi(x)$ be the first eigenvalue and corresponding eigenfunction of the eigenvalue problem as follows:

$$
-\Delta \phi(x)=\lambda \phi(x), \quad x \in \Omega ; \quad \phi(x)=0, \quad x \in \partial \Omega .
$$

Then $\lambda_{1}>0$. Standardized $\phi(x)$, such that $\left.\phi(x)\right|_{\Omega}>0$ and $\max _{x \in \Omega} \phi(x)=1$, then $\left.(\partial \phi / \partial n)\right|_{\partial \Omega}<0$, where $n$ is the outer normal direction of $\partial \Omega$, suppose $\Omega_{1} \subset \subset \Omega$ is a compact subset of $\Omega$, if any solution $(u, v, w)$ blows up in $\Omega_{1}$, also $(u, v, w)$ blows up in $\Omega$.

Define functions

$$
\begin{gathered}
\underline{u}(x, t)=(s(t) \phi(x))^{l_{1}}, \quad \underline{v}(x, t)=(s(t) \phi(x))^{l_{2}}, \\
\underline{w}(x, t)=(s(t) \phi(x))^{l_{3}},
\end{gathered}
$$

where $l_{1}, l_{2}$, and $l_{3}$ satisfy $m l_{1}, n l_{2}, h l_{3}>1$, and $s(t)$ is the solution of the initial value problem as follows:

$$
s^{\prime}(t)=k s^{d}, \quad s(0)=b .
$$

Here $k, b>0, d>1$ is to be determined suitably. It is clear that $s(t) \geq b$ and $s(t)$ are unbounded in finite time, and

$$
\begin{array}{r}
\underline{u}(x, t) \geq(\rho b)^{l_{1}}>0, \\
\underline{v}(x, t) \geq(\rho b)^{l_{2}}>0, \\
\underline{w}(x, t) \geq(\rho b)^{l_{3}}>0, \\
x \in \Omega_{1}, \quad t>0,
\end{array}
$$

where $\rho=\min _{\bar{\Omega}} \phi(x)>0$; by computing we obtain that

$$
\begin{aligned}
\Delta \underline{u}^{m}+\underline{u}^{p_{1}}\|\|_{\alpha}^{p_{2}}= & s^{m l_{1}}\left[m l_{1}\left(m l_{1}-1\right) \phi^{m l_{1}-2}|\nabla \phi|^{2}\right. \\
& \left.+m l_{1} \phi^{m l_{1}-1} \Delta \phi\right] \\
& +s^{l_{1} p_{1}+l_{2} p_{2}} \phi^{l_{1} p_{1}}\left\|\phi^{l_{2}}\right\|_{\alpha}^{p_{2}} \\
\geq & -\lambda_{1} m l_{1} s^{m l_{1}} \phi^{m l_{1}}+C_{1} s^{l_{1} p_{1}+l_{2} p_{2}}
\end{aligned}
$$

$$
\begin{gathered}
=l_{1} s^{l_{1}-1} \phi^{l_{1}}\left(-\lambda_{1} m s^{m l_{1}-l_{1}+1} \phi^{m l_{1}-l_{1}}\right. \\
\left.+\frac{C_{1} s^{l_{1} p_{1}+l_{2} p_{2}-l_{1}+1} \phi^{-l_{1}}}{l_{1}}\right) \\
\geq l_{1} s^{l_{1}-1} \phi^{l_{1}} \frac{C_{1}}{l_{1}} s^{m l_{1}-l_{1}+1} \\
\times\left(-\frac{\lambda_{1} m l_{1}}{C_{1}}+s^{l_{1} p_{1}+l_{2} p_{2}-m l_{1}}\right), \\
\underline{u}_{t}=l_{1} s^{l_{1}-1} \phi^{l_{1}} s^{\prime}(t) .
\end{gathered}
$$

Similarly

$$
\begin{gathered}
\Delta \underline{v}^{n}+\underline{v}^{q_{1}}\|\underline{w}\|_{\beta}^{q_{2}} \\
\geq l_{2} s^{l_{2}-1} \phi^{l_{2}} \frac{C_{2}}{l_{2}} s^{n l_{2}-l_{2}+1}\left(-\frac{\lambda_{1} n l_{2}}{C_{2}}+s^{l_{2} q_{1}+l_{3} q_{2}-n l_{2}}\right), \\
\underline{v}_{t}=l_{2} s^{l_{2}-1} \phi^{l_{2}} s^{\prime}(t), \\
\Delta \underline{w}^{h}+\underline{w}^{r_{1}}\|\underline{u}\|_{\gamma}^{r_{2}} \\
\geq l_{3} s^{l_{3}-1} \phi^{l_{3}} \frac{C_{3}}{l_{3}} s^{h l_{3}-l_{3}+1}\left(-\frac{\lambda_{1} h l_{3}}{C_{3}}+s^{l_{3} r_{1}+l_{1} r_{2}-h l_{3}}\right), \\
\underline{w}_{t}=l_{3} s^{l_{3}-1} \phi^{l_{3}} s^{\prime}(t),
\end{gathered}
$$

where

$$
\begin{gathered}
C_{1} \equiv \phi^{l_{1} p_{1}}\left\|\phi^{l_{2}}\right\|_{\alpha}^{p_{2}}, \quad C_{2} \equiv \phi^{l_{2} q_{1}}\left\|\phi^{l_{3}}\right\|_{\beta}^{q_{2}}, \\
C_{3} \equiv \phi^{l_{3} r_{1}}\left\|\phi^{l_{1}}\right\|_{\gamma}^{r_{2}} .
\end{gathered}
$$

(1) If $m>p_{1}, n>q_{1}, h>r_{1}$, and $p_{2} q_{2} r_{2}>\left(m-p_{1}\right)(n-$ $\left.q_{1}\right)\left(h-r_{1}\right)$, there exist positive constants $l_{1}, l_{2}$, and $l_{3}$ satisfying $m l_{1}, n l_{2}, h l_{3}>1$ and

$$
\begin{gathered}
p_{2} l_{2}>\left(m-p_{1}\right) l_{1}, \quad q_{2} l_{3}>\left(n-q_{1}\right) l_{2}, \\
r_{2} l_{1}>l_{3}\left(h-r_{1}\right) .
\end{gathered}
$$

Choose

$$
\begin{gathered}
k=\min \left\{\frac{C_{1}}{l_{1}}, \frac{C_{2}}{l_{2}}, \frac{C_{3}}{l_{3}}\right\}, \\
d=\min \left\{m l_{1}-l_{1}+1, n l_{2}-l_{2}+1, h l_{3}-l_{3}+1\right\}, \\
b=\max \left\{\left(1+\frac{\lambda_{1} m l_{1}}{C_{1}}\right)^{1 /\left(l_{1} p_{1}+l_{2} p_{2}-m l_{1}\right)},\right. \\
\left(1+\frac{\lambda_{1} n l_{2}}{C_{2}}\right)^{1 /\left(l_{2} q_{1}+l_{3} q_{2}-n l_{2}\right)}, \\
\left.\left(1+\frac{\lambda_{1} h l_{3}}{C_{3}}\right)^{1 /\left(l_{3} r_{1}+l_{1} r_{2}-h l_{3}\right)}\right\} .
\end{gathered}
$$

From (26)-(27) and $m, n, h>1$, we have $k>0, d>1$, and $b>1$. Suppose that $u_{0}, v_{0}$, and $w_{0}$ are sufficiently large to satisfy

$$
\begin{gathered}
u_{0}(x) \geq(b \phi)^{l_{1}}, \quad v_{0}(x) \geq(b \phi)^{l_{2}}, \\
w_{0}(x) \geq(b \phi)^{l_{3}} .
\end{gathered}
$$


From $(20)-(28)$, we obtain that $(\underline{u}, \underline{v}, \underline{w})$ is positive weak lower-solutions of system (1) in $\Omega_{1}$.

(2) If $m \leq p_{1}, n \leq q_{1}$, or $h \leq r_{1}$, (26) still holds, the results are obtained by the same methods.

Thus completes the proof of Theorem 2 .

\section{Conflict of Interests}

The author declares that there is no conflict of interests regarding the publication of this paper.

\section{Acknowledgments}

The author would like to thank the referees for their helpful comments and suggestions which greatly improve the presentation of the paper. This work was supported by the Program for Science and Technology Development Foundation of Fujian Education Bureau (JA12374).

\section{References}

[1] C. C. Wu, "Global existence of positive solution for a nonlinear parabolic system," Journal of Fuqing Branch of Fujian Normal University, no. 2, pp. 22-24, 2012.

[2] C. C. Wu, "The blow-up property of positive solutions to a class of nonlinear parabolic equations with cross-coupling," Journal of Fujian University of Technology, vol. 10, no. 6, pp. 533-535, 2012.

[3] C. C. Wu, "Solvability of a nonlinear parabolic system," Journal of Putian University, vol. 19, no. 2, pp. 25-27, 2012.

[4] Y.-j. Chen and Y.-p. Zhu, "Blow-up rate and profile for a class of quasilinear parabolic system," Applied Mathematics and Mechanics, vol. 30, no. 7, pp. 811-820, 2009.

[5] H. L. Li, "Blow-up estimates for semilinear parabolic systems with exponent reaction terms," Journal of Southeast University, vol. 39, no. 6, pp. 1287-1291, 2009.

[6] Y. Z. Xue, "Global existence and blow-up problem of a class of nonlinear parabolic equations with cross coupling," Journal of Lanzhou University of Technology, vol. 37, no. 4, pp. 162-164, 2011.

[7] Y. J. Wei and J. Wu, "Existence and blow-up of solutions to a quasilinear degenerate parabolic system," Journal of Jilin University, vol. 48, no. 3, pp. 406-408, 2010.

[8] X. S. Wu and W. J. Gao, "Global existence and blow-up of solutions to an evolution $p$-Laplace system with nonlinear boundary conditions," Journal of Jilin University, vol. 47, no. 6, pp. 11051111, 2009.

[9] Y. P. Chen and L. H. Liu, "Global blow-up for a localized nonlinear parabolic equation with a nonlocal boundary condition," Journal of Mathematical Analysis and Applications, vol. 384, no. 2, pp. 421-430, 2011.

[10] Y. J. Chen, "Blow-up and global existence for a nonlocal degenerate quasilinear parabolic system," Acta Mathematica Scientia A, vol. 30, no. 2, pp. 386-396, 2010. 


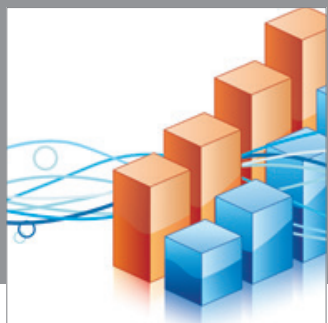

Advances in

Operations Research

mansans

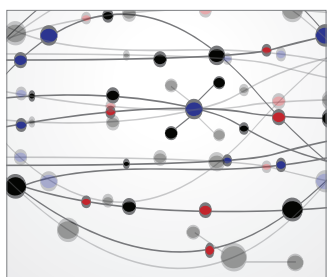

The Scientific World Journal
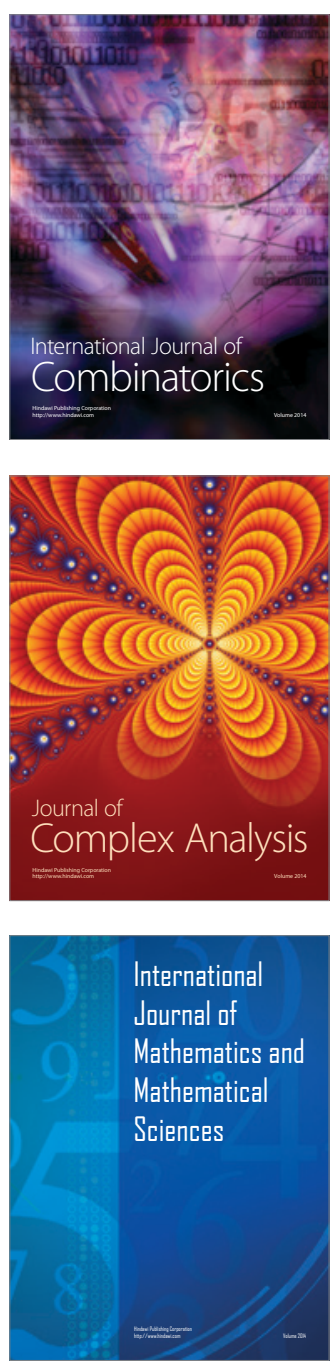
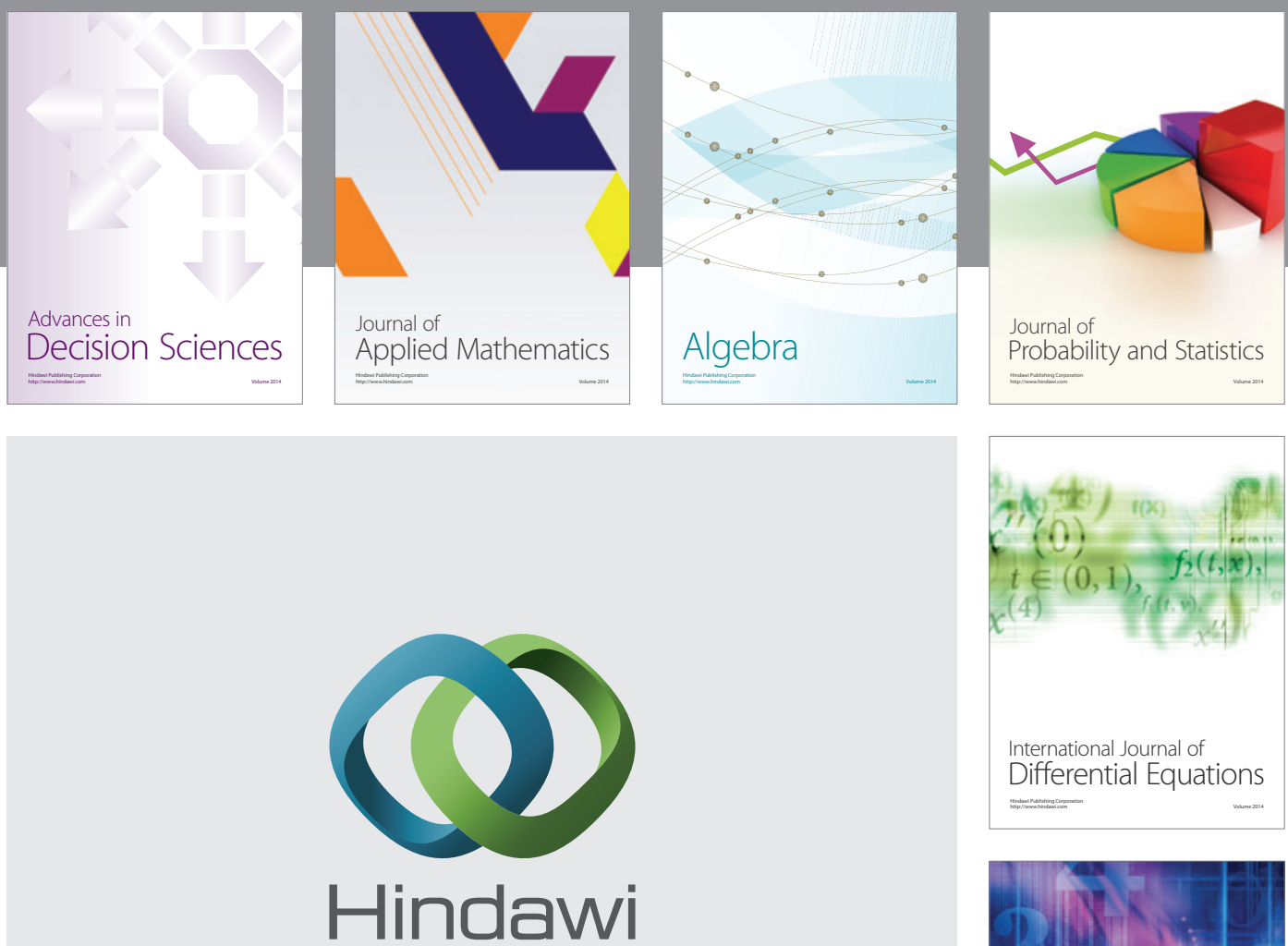

Submit your manuscripts at http://www.hindawi.com
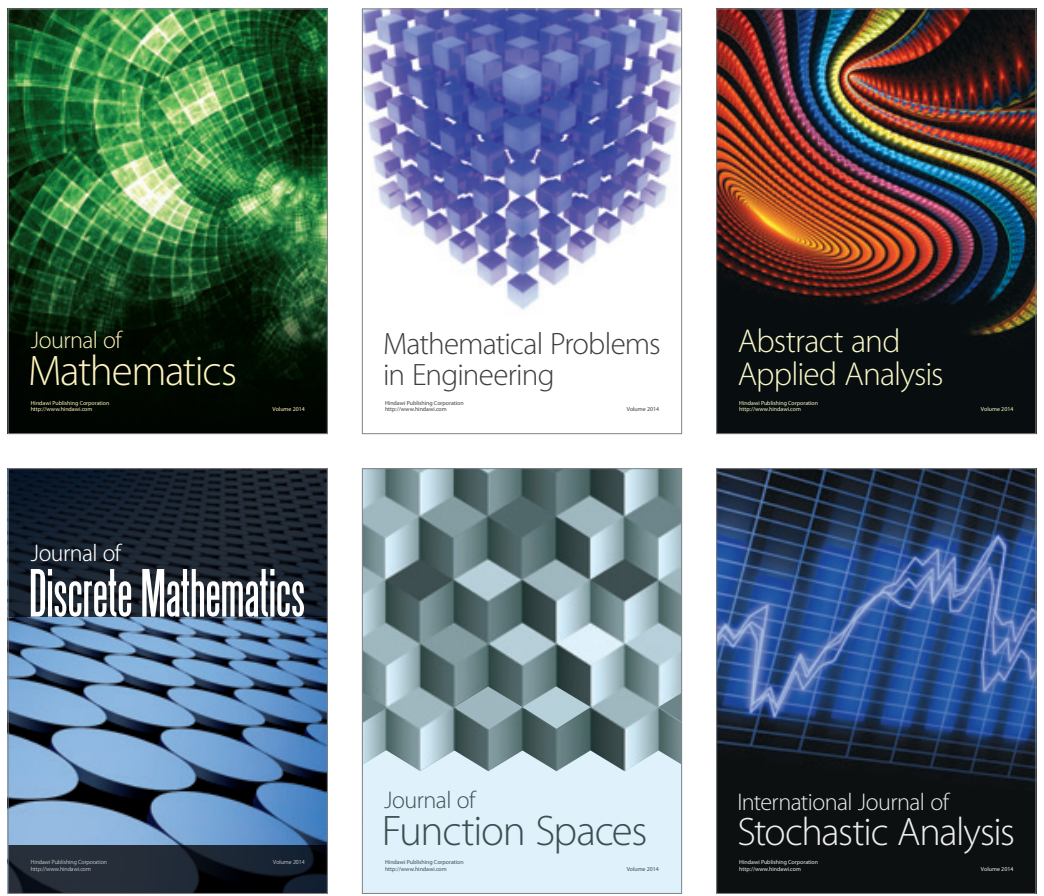

Journal of

Function Spaces

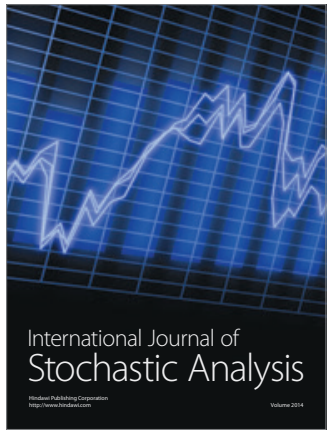

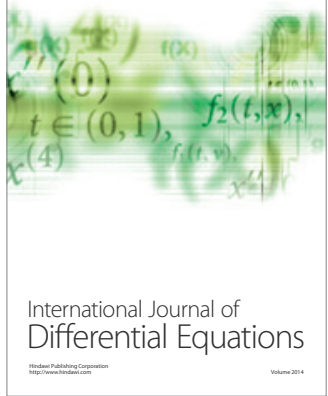
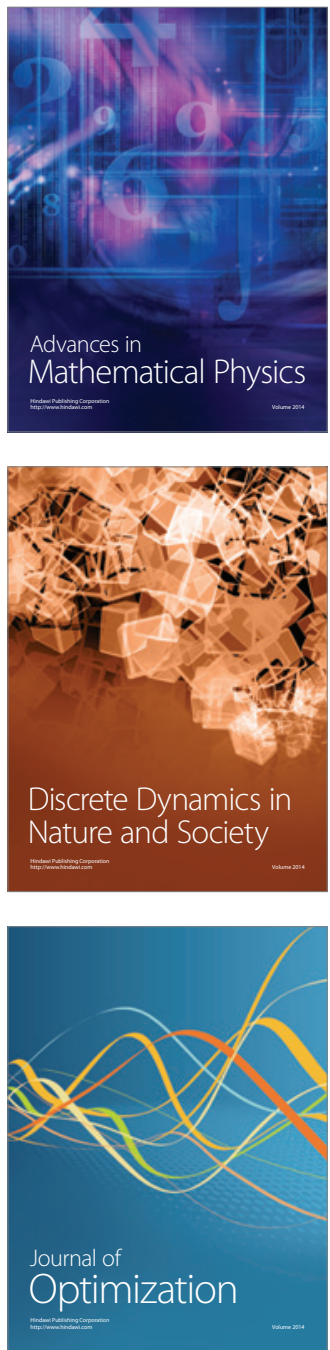